León-Sánchez, R., y Barrera-García, K. (enero-abril, 2022). Enfoques y estilos de aprendizaje en estudiantes de Psicología de una universidad pública en México. Revista Virtual Universidad

Católica del Norte, (65), 102-136. https://www.doi.org/10.35575/rvucn.n65a5

\title{
Enfoques y estilos de aprendizaje en estudiantes de psicología de una universidad pública en México
}

\begin{abstract}
Approaches and learning styles in Psychology students of a public university in Mexico
\end{abstract}
\section{Rigoberto León-Sánchez}

Doctor en Psicología Educativa y del Desarrollo

Facultad de Psicología, Universidad Nacional Autónoma de México.

Ciudad de México, México

rigobert@unam.mx

Orcid: https://orcid.org/0000-0002-0838-3507

\section{Kirareset Barrera-García}

Doctora en Psicología Educativa y del Desarrollo

Facultad de Psicología, Universidad Nacional Autónoma de México.

Ciudad de México, México

kirareset@gmail.com

Orcid: https://orcid.org/0000-0002-2327-5571

Recibido: 17 de febrero de 2021

Evaluado: 9 de septiembre de 2021

Aprobado: 7 de diciembre de 2021

Tipo de artículo: Investigación Científica y Tecnológica

\section{Resumen}

Con el objetivo de examinar los estilos y enfoques de aprendizaje, se aplicaron dos cuestionarios (CHAEA y R-SPQ-2F) a estudiantes de psicología $(N=221)$ de tres semestres diferentes. Los resultados obtenidos mostraron que el estilo Reflexivo fue elegido en primer lugar, mientras que el estilo Activo lo fue en último $(F(2.071,455.620)=45.836, p<.001)$. No se encontraron diferencias entre los tres semestres examinados. Por otro lado, los participantes se decantaron por el enfoque Profundo de aprendizaje y no por el Superficial $(t(220)=24.603, p<.001, d$ de Cohen 
= 2.55). Una prueba de Pearson mostró relaciones fuertes y positivas entre el enfoque Profundo y los estilos de aprendizaje Reflexivo, Teórico y Pragmático. Tal y como se ha encontrado en otros estudios, los participantes se inclinaron por el estilo Reflexivo y eligieron el enfoque Profundo, lo cual parece reflejar la forma en cómo se desarrolla el trabajo académico realizado en el nivel universitario. Es importante mencionar que las características psicométricas de los instrumentos utilizados poseen una consistencia adecuada, lo cual sustenta su empleo para el análisis de los estilos y enfoques de los estudiantes como un paso previo antes de su inserción en el proceso educativo.

Palabras clave: Educación superior; Enfoques de aprendizaje; Estilos de aprendizaje; Estudiantes de psicología; Estudiantes universitarios.

\begin{abstract}
In order to examine the styles and approaches of, two questionnaires (CHAEA and R-SPQ-2F) were applied to psychology students, $(\mathrm{N}=221)$, from three different semesters. The results obtained showed that the Reflective style was chosen in the first place while the Active style was chosen last, $F(2,071,455,620)=45,836, p<.001$. No differences were found between the three semesters examined. On the other hand, the participants opted for the Deep learning approach and not for the Superficial one, $t(220)=24.603, p<.001$, Cohen's $d=2.55$. A Pearson test showed strong and positive relationships between the Deep approach and the Reflective, Theoretical and Pragmatic learning styles. As has been found in other studies, our participants favored the Reflective style and chose the Deep approach, which seems to reflect the way in which academic work carried out at university level develops. It is important to mention that psychometric characteristics of the instruments used have an adequate consistency, which supports their use for the analysis of the styles and approaches of the students as a previous step before their insertion in the educational process.
\end{abstract}

Keywords: Higher education; Approaches learning; Learning styles; Psychology students; Graduate students. 
Enfoques y estilo de aprendizaje | Revista Virtual

Universidad Católica del Norte, 65, 102-136

ISSN: 0124-5821 (En línea)

\section{Introducción}

De acuerdo con Alexander et al. (2009), el aprendizaje es un proceso multidimensional que produce un cambio relativamente permanente en un estudiante. Un cambio que, básicamente, repercute en cómo percibe su entorno y cómo responde a este. Sin embargo, el proceso de aprendizaje no se despliega en el vacío; es un proceso dinámico entre el estudiante, el profesor y el objeto del aprendizaje, y tiene como escenario un contexto espacio-temporal determinado (Osher et al., 2020). En este sentido, el proceso puede ser afectado por factores tales como la actividad mental de los estudiantes, su nivel de desarrollo, la dinámica de la institución, su cultura, su concepción de "eficacia escolar", las políticas educativas, sus emociones, entre otros (Cimermanová, 2018; Cornejo y Redondo, 2007; Karagiannopoulou et al., 2020; Rosário, Núñez, González-Pienda, Almeida et al., 2005; Rico-Reintsch, 2019; Rosário, Núñez, González-Pienda, Valle et al., 2010).

Específicamente, en cuanto a los factores intrínsecos al estudiante, existe evidencia de que en el proceso de aprendizaje no solo interactúan las representaciones actuales y pasadas que ha construido acerca del mundo, sino que dicha interacción se ve afectada por los cambios que ocurren como resultado del desarrollo psicológico (Osher et al., 2020), la motivación, las demandas y estructura del contexto en que se encuentra, así como por la manera como enfrenta dicho proceso (López-Vargas et al., 2011); ya sea por su estilo de aprendizaje (Alonso et al., 1994; AltamiranoDroguett et al., 2019; Bravo et al., 2020; Cassidy, 2004; Dunn, 1984; Gómez et al., 2017; Kolb \& Kolb, 2005a), o por su manera de enfocarlo o de aproximarse a él (Biggs, 1987; Duff \& McKinstry, 2007; Entwistle, 1991; Freiberg-Hoffmann, Berenguer et al., 2017; Freiberg-Hoffmann y Fernández-Liporace, 2016; Leiva-Brondo et al., 2020; López y López, 2013).

En pocas palabras, el aprendizaje no solo está determinado por la capacidad cognitiva del estudiante, su historia académica, deseos y necesidades, sino también por sus preferencias y tendencias para aprender cómo lo hace; es decir, por el modo habitual y distintivo que tiene de adquirir conocimiento (McLoughlin, 1999) y de concebirlo (Alexander et al., 2009; Dantas \& Cunha, 2020; Fryer \& Vermunt, 2018; Patiño-Toro et al., 2020; Valle et al., 2000).

El constructo estilo de aprendizaje ha sido utilizado por las aproximaciones centradas en la cognición y por las aproximaciones centradas en el aprendizaje (Cassidy, 2004); y si bien se han 
instrumentado diversos medios con el propósito de medirlo, por ejemplo, el Cuestionario HoneyAlonso de Estilos de Aprendizaje (CHAEA) de Alonso et al. (1994), el Learning Style Inventory (LSI) de Kolb (Kolb \& Kolb, 2005b) y el Learning Style Questionnaire (LSQ) de Honey y Mumford (véase Duff \& Duffy, 2002), parece haber acuerdo entre los investigadores en definir estilo de aprendizaje como lo hace Keefe (como se citó en Reid, 1987); a saber, son un conjunto de rasgos cognitivos, afectivos y fisiológicos relativamente estables que prescriben cómo los discentes perciben, interactúan y responden a sus ambientes de aprendizaje. Por ende, la manera en la cual un estudiante se aproxima a una situación de aprendizaje no solo impacta en la ejecución y en el resultado de aprendizaje (Cassidy, 2004), sino también en las estrategias que utilice (Abalde et al., 2009; Rivera et al., 2013; Demirbas \& Demirkan, 2007).

Los estilos de aprendizaje implican la personalidad (características individuales), las formas de procesar la información por diferentes canales perceptivos (habilidades cognitivas) y las maneras de afrontar, resolver y actuar en el mundo (comportamiento). Si bien se asume que los estilos de aprendizaje son relativamente estables en un individuo, es posible que sufran cambios; por ejemplo, que sean diferentes en situaciones y contextos diferentes (Demirbas \& Demirkan, 2007; De Vita, 2001; Gómez et al., 2017; Rodríguez et al., 2018; Rojas-Jara et al., 2016), o bien, que sean susceptibles de mejorar.

Un aspecto práctico de la investigación sobre estilos de aprendizaje ha llevado a considerar que los responsables de organizar el proceso de enseñanza-aprendizaje deberían de tomar en cuenta las diferencias individuales de los aprendices (aspectos cognitivos, elementos de la personalidad y formas específicas de relacionarse con el medio), dado que los estilos de aprendizaje son afectados, positiva o negativamente, por una determinada forma de enseñar (Landrum \& McDuffie, 2010; Martínez et al., 2019; Rojas-Jara et al., 2016). De este modo, es posible que, si se les enseña a los estudiantes de acuerdo con su propio estilo de aprendizaje, se maximice y torne más efectivo el proceso de aprendizaje (Adán, 2008).

Una línea de investigación íntimamente relacionada con aquella que tiene como objeto los estilos de aprendizaje es la que indaga la manera que tienen los estudiantes de enfocarse o aproximarse al aprendizaje (Justicia et al., 2008); la misma puede variar dependiendo de los contenidos a aprender, el contexto y las demandas específicas de la tarea (Díaz-García et al., 2020; Karagiannopoulou et al., 2020; Monroy y Hernández Pina, 2014; Richardson, 2010). 
Como lo señalan Valle et al. (2000), la investigación sobre los enfoques de aprendizaje examina las percepciones que tienen los estudiantes de las tareas académicas, asumiendo que dichas percepciones están orientadas tanto por los motivos y las metas como por las estrategias que se ponen en juego para cumplimentar la tarea de aprendizaje (Abalde et al., 2009; Astika \& Sumakul, 2020; Bana \& Rizvi, 2019; Biggs, 1987; Rosário, Núñez, González-Pienda, Valle et al., 2010). La evidencia indica que, al menos, existen dos enfoques o aproximaciones de aprendizaje: una aproximación profunda, encaminada a la comprensión y una aproximación superficial enfocada a la simple reproducción del material (Entwistle, 1991; Duff \& McKinstry, 2007; Freiberg-Hoffmann y Fernández-Liporace, 2016; López y López, 2013). Aunque algunos investigadores (Kember, 2000) proponen también la existencia de una aproximación equilibrada, la cual se caracterizaría por el doble propósito del estudiante de comprender y memorizar. De acuerdo con Duff \& McKinstry (2007), quienes estudian la aproximación de los estudiantes al aprendizaje tienden a centrarse más en analizar la interacción entre el aprendiz y el ambiente en el cual se desarrolla el proceso de aprendizaje que en el análisis de las preferencias o estilos de aprendizaje.

De acuerdo con una revisión de las investigaciones sobre los estilos de aprendizaje llevadas a cabo en países iberoamericanos (Bahamón et al., 2012), la mayoría de estas se han realizado con estudiantes de nivel universitario y utilizan el Cuestionario CHAEA (Alonso et al., 1994). Los resultados del trabajo de validación y fiabilidad del cuestionario (Alonso et al., 1994) indican valores aceptables para los cuatro estilos de aprendizaje: Activo ( $\alpha=.627)$, Reflexivo $(\alpha=.727)$, Teórico $(\alpha=.658)$ y Pragmático $(\alpha=.585)$. Asimismo, los índices de correlación encontrados entre los distintos estilos muestran una relación positiva fuerte entre Reflexivo y Teórico, pero una relación negativa entre Activo y Reflexivo. Estos datos, y los obtenidos en otras investigaciones en las cuales se ha utilizado y/o validado el CHAEA (Alonso y Gallego, 2010; Freiberg-Hoffmann, Abal y Fernández-Liporace, 2020; Juárez, 2014; Santizo et al. 2008), permiten asumir que es un cuestionario con buenas propiedades psicométricas (López-Aguado \& Gutiérrez-Provecho, 2018; Vergara-Hernández et al., 2019; Zakariya et al., 2020).

Igualmente, en el grueso de los estudios analizados se ha reportado que el estilo de aprendizaje marcadamente encontrado es el Reflexivo. Esta tendencia no parece variar por las carreras que cursan los estudiantes ni por el contexto cultural. Por ejemplo, los resultados 
obtenidos por Gil Madrona et al. (2007) señalan que $62 \%$ de la muestra examinada, de estudiantes de magisterio de una universidad española, se decantó por el estilo Reflexivo. El mismo dato lo obtuvieron Ordoñez et al. (2003) con estudiantes de ciencias de la salud de la Universidad de Cádiz, así como Ortiz y Canto (2013) con estudiantes de ingeniería de una universidad mexicana, Altamirano-Droguett et al. (2019) con estudiantes de obstetricia, Esguerra y Guerrero (2010) con estudiantes de psicología o Bravo et al. (2020) con estudiantes de enfermería.

Con todo, si bien en algunas investigaciones, como en el estudio de Camarero et al. (2000), se encontró que los estudiantes universitarios de física, matemáticas, derecho e informática tendieron hacia los estilos de aprendizaje Reflexivo y Teórico, eso no ocurrió en todos los casos, por ejemplo, los estudiantes de la Diplomatura de Magisterio utilizaron mayormente el estilo Activo. Autores como Gómez et al. (2017) han encontrado resultados similares con estudiantes universitarios de educación a distancia; solo que en este caso predominan los estilos de aprendizaje activo y teórico en los estudiantes de primer semestre, mientras que en el décimo semestre predominan los estilos reflexivo y pragmático. Por último, un estudio llevado a cabo con estudiantes de medicina de cuatro universidades latinoamericanas y dos españolas (Díaz-Véliz et al., 2009), utilizando tanto el CHAEA como el LSI, encontró que mientras los estudiantes diferían entre los estilos Acomodador, Divergente y Asimilador de Kolb \& Kolb (2005b), todos ellos tendieron hacia el estilo de aprendizaje Reflexivo (Alonso et al., 1994).

Por otra parte, en las investigaciones sobre los enfoques de aprendizaje, los datos obtenidos muestran un panorama más diverso. Se ha encontrado que estudiantes de enfermería e ingeniería tienden marcadamente hacia el enfoque Profundo, a diferencia de los estudiantes de organización deportiva en quienes no se observa esta tendencia (Esquivel et al., 2009). No obstante, otra investigación (Hernández Pina et al. 2010) encontró que los estudiantes de ciencias del deporte, de tres diferentes universidades españolas y una mexicana, tienden, significativamente, hacia el enfoque Profundo. Lo mismo sucede con estudiantes de ciencias de la vida, quienes se decantan más por una aproximación profunda que por una superficial (Leiva-Brondo, et al., 2020), de manera similar a como lo hacen estudiantes de medicina (Mansfield et al., 2020). Por último, en algunos casos (Buendía y Olmedo, 2003) se ha encontrado que estudiantes españoles y argentinos eligen distintos enfoques: superficial, los primeros, y Profundo, los segundos. Recio (2008) encontró que estudiantes universitarios de psicología y educación social de la Universidad 
Nacional de Educación a Distancia, sede Sevilla, de primer ingreso, adoptaban el enfoque Profundo en una gran proporción.

Algunos trabajos han examinado las relaciones existentes entre los estilos de aprendizaje (CHAEA) y otros constructos, como las estrategias de aprendizaje (Juárez et al., 2016) o la autoeficacia (Laffita y Guerrero, 2017). Otros han analizado las relaciones entre los enfoques y los estilos de aprendizaje (Cuthbert, 2005; Freiberg-Hoffmann, Abal y Fernández-Liporace, 2020; Freiberg-Hoffmann, Berenguer et al., 2017; Richardson, 2011) y resaltado la importancia de este tipo de estudios para aumentar la comprensión sobre el proceso de aprendizaje, y de este modo, servirse de esos resultados para mejorar las formas de enseñanza (Richardson, 2005).

Este conjunto de contrastes solo muestra lo complejo que es la red de variables que se entrecruzan en la práctica educativa; una práctica que es mejor descrita como una actividad cognitiva constructiva autorregulada que implica un aprendizaje complejo (Castañeda, 2004; Kirschner \& Van Merriënboer, 2008). De acuerdo con Castañeda y Ortiz (2017), el aprendizaje complejo está influido por poderosos componentes de agencia académica, entre ellos la epistemología personal, un componente que parece influir en todo el proceso. Las creencias epistemológicas han sido generalmente conceptualizadas como concepciones que tienen los individuos sobre el conocimiento y el conocer (Hofer \& Sinatra, 2010), implicando, asimismo, creencias sobre la naturaleza y adquisición del conocimiento. En su estudio, Paulsen \& Feldman (2005) encontraron que los estudiantes con creencias epistemológicas basadas en conocimiento simple (por ejemplo, considerar que el conocimiento está organizado en piezas o fragmentos), generalmente utilizan menos estrategias motivacionales autorreguladas. Un hallazgo importante de este estudio fue que aquellos estudiantes que poseen creencias epistemológicas más sofisticadas consideran que la capacidad de aprendizaje puede ser mejorada, mientras que para los estudiantes con creencias epistemológicas ingenuas el aprendizaje es una habilidad fija y solo responden a las demandas escolares, en lugar de desarrollar una capacidad de autorregulación del aprendizaje. Otros autores han señalado que las creencias epistemológicas de los estudiantes universitarios no solo se relacionan con sus ideas sobre el proceso de aprendizaje, sino que también influyen en toda su trayectoria académica (Chan, 2003; Hofer, 2004; Lonka et al., 2021; Mohamed \& El-Habbal, 2013; Schommer, 1998; Tanriverdi, 2012). Por ejemplo, se ha encontrado un vínculo entre las creencias epistemológicas y las metas de logro (Winberg et al., 2019), las habilidades de 
argumentación (Hofer \& Pintrich, 1997), las prácticas docentes (García y Sebastián, 2011), las habilidades metacognitivas y de autorregulación (Hofer \& Sinatra, 2010) y los enfoques de aprendizaje (Phan, 2008).

Es probable, como lo señalan Brownlee et al. (2017), que el papel de las creencias epistemológicas sea sutil, pero omnipresente. A saber, es probable que estas creencias influyan en cómo aprenden los estudiantes, cómo instruyen los maestros y, posteriormente, cómo los maestros modifican, consciente o inconscientemente, las creencias epistemológicas de los estudiantes.

En el caso particular, el propósito del presente trabajo fue examinar, en un grupo de estudiantes de la carrera de psicología de una universidad de la Ciudad de México, tanto sus estilos de aprendizaje como la manera en la cual se aproximan al aprendizaje, así como también las posibles relaciones entre ambos constructos, lo cual puede iluminar de mejor manera la complejidad de la práctica educativa.

\section{Método}

El presente estudio fue de corte no experimental y tuvo como objetivo conocer el estilo y el enfoque de aprendizaje de los estudiantes de psicología.

\section{Participantes}

Se seleccionaron 221 estudiantes de la carrera de psicología (177 mujeres y 44 hombres), quienes cursaban segundo $(n=23)$, cuarto $(n=126)$ y sexto $(n=72)$ semestre. Las edades de los participantes tenían un rango entre 18 y 28 años $(M=20.91, D E=2.43)$. Respecto de los sistemas escolares en los cuales cursaban la carrera, 32 de los participantes pertenecían al Sistema de Universidad Abierta (SUA) y 189 al Sistema Escolarizado (SE). El promedio de calificaciones reportado por los participantes, hasta el momento de la encuesta, se distribuyó de 7.00 a 10.00 ( $M$ $=8.81, D E=.530)$. A partir de la distribución cuartilar, se dividió a los participantes en dos grupos, a partir del percentil 50. G1 Promedio, de 7.30 a 8.79 y G2 Promedio, de 8.80 a 10.0. Las variables para contrastar fueron semestre y promedio. 


\section{Instrumentos}

Cuestionario Honey-Alonso de Estilos de Aprendizaje (CHAEA). Este cuestionario consta de 80 ítems, calificados como positivos o negativos, y organizados en cuatro grupos de 20 ítems, cada uno correspondiente a los cuatro estilos de aprendizaje: Activo (ACT), Reflexivo (REF), Teórico (TEO) y Pragmático (PRA) (Juárez, 2014). El estilo Activo define a los estudiantes como participativos, vitales, animadores, deseosos de aprender y voluntariosos, entre otras características. Les gusta actuar y experienciar nuevas situaciones. El Reflexivo, incluye a estudiantes que son concienzudos, observadores, analíticos, inquisidores, detallistas y prudentes. Por ello les gusta analizar con detenimiento los datos que se les presentan (Arenas, 2017). El estilo Teórico describe a los estudiantes como metódicos, disciplinados, a quienes les gusta pensar, ser sistemáticos. Les gusta la teoría y la racionalidad que de ella emana, su lógica. Por último, el estilo Pragmático, define a los estudiantes que tienden a probar teorías o técnicas nuevas, intentando comprobar si funcionan en la práctica. Por ende, y dado que son prácticos y apegados a la realidad, les desagradan las divagaciones sobre un mismo tema (Alonso et al., 1994; Ortiz y Canto, 2013).

Para el presente estudio, fueron revisados cada uno de los 80 ítems que conforman el cuestionario. Los pocos cambios que se hicieron fueron respecto de la redacción y el uso de los vocablos. Por ejemplo, el ítem 3, el cual originalmente dice: "Muchas veces actúo sin mirar las consecuencias", fue cambiado por "Muchas veces actúo sin pensar en las consecuencias"; esta sería mejor comprendida. El segundo cambio fue respecto de la calificación de los ítems; en lugar de ser calificados como positivos o negativos (escala dicotómica) lo fueron en una escala Likert de cuatro puntos: $1=$ Totalmente en desacuerdo, $2=$ En desacuerdo, $3=$ De acuerdo y $4=$ Totalmente de acuerdo.

Study Processs Questionnaire revisado de dos factores (R-SPQ-2F) de Biggs et al. (2001; véase también Justicia et al., 2008). El R-SPQ-2F es una versión reducida del Study Process Questionnaire (Biggs, 1987), originalmente compuesto por 42 ítems y organizado en tres escalas: Aproximación Profunda, Aproximación Superficial y Aproximación al Logro. Cada escala, a su vez, se organiza en dos sub-escalas: Motivación y Estrategia. El R-SPQ-2F está compuesto únicamente por dos escalas, de 10 ítems cada una de ellas (Profunda y Superficial [AP y AS, respectivamente]) y las mismas dos sub-escalas (Motivación y Estrategia), quedando así cuatro 
sub-escalas (compuestas por 5 ítems cada una), las cuales reflejan aspectos esenciales de la motivación y de las estrategias utilizadas en el proceso de aprendizaje. Motivación superficial (MS) es concebida como una motivación instrumental y cuyo principal propósito es obtener una buena calificación, sin más aspiraciones que la aprobación de las asignaturas, y con su correspondiente miedo al fracaso. En contraparte, motivación profunda (MP) es intrínseca, el estudio está dirigido por el interés de los estudiantes en temas académicos particulares. Por el otro lado, mientras que la estrategia superficial (ES) es reproductiva y el acto de aprender, para los estudiantes, tiene por objetivo encontrar lo esencial de los temas y reproducirlo a través del aprendizaje memorístico, la estrategia profunda (EP) está dirigida a la búsqueda del significado, el aprendiz se esfuerza por hacer que los contenidos se relacionen pertinentemente con sus conocimientos anteriores.

El trabajo de validez y confiabilidad realizado con el R-SPQ-2F fue llevado a cabo con 495 estudiantes universitarios de varias disciplinas (Biggs et al., 2001). La aproximación profunda obtuvo una $\alpha=0.73$ y la aproximación superficial una $\alpha=0.64$. Por su parte las cuatro sub-escalas obtuvieron valores Alfa bastante aceptables (0.62; 0.63; 0.72 y 0.57 para MP, EP, MS y ES, respectivamente). Asimismo, pudo observarse que el comportamiento de los 20 ítems que componen la versión final es consistente y congruente, en cuanto a su pertenencia a las dos aproximaciones (AP 10 ítems; AS 10 ítems), así como bien diferenciados respecto de la manera en que reflejan las características específicas de los componentes motivación y estrategia en cada una de las aproximaciones. Por todo ello, el R-SPQ-2F posee apropiadas características psicométricas y parece ser muy útil para identificar la forma en la que los estudiantes se aproximan al proceso de aprendizaje.

Para estructurar el instrumento, el cuestionario, junto con el Manual (Biggs, 1987), fueron enviados a una traductora profesional. Cuando se tuvo la traducción de todos los elementos que componían el cuestionario (instrucciones, ítems, entre otros) se revisaron cada uno de ellos en un seminario sobre investigación educativa que coordinaba el primer autor de este estudio. En dicho seminario, y habiendo revisado con anterioridad las investigaciones de Biggs y sus colaboradores sobre las aproximaciones al aprendizaje y la enseñanza, se realizó una revisión de la traducción. Específicamente, fue discutida y comentada la traducción de cada uno de los 20 ítems, poniendo 
especial énfasis en si concordaba no solo con el original en inglés, sino además si conservaba el sentido de pertenencia a cada una de las sub-escalas.

Cuando fue necesario, en el seminario se propuso una nueva versión o bien una adecuación de términos que, en opinión de los estudiantes, se entendía mejor en español. Terminado esta fase, le fue regresado a la traductora la nueva versión para que ella hiciera los arreglos pertinentes y considerara si era válido el arreglo a la traducción. Por último, se cotejó la versión final con la versión adaptada al español de Freiberg-Hoffmann, Abal y Fernández-Liporace (2020), viendo que no había grandes diferencias entre dicha versión y la que se elaboró en el seminario. Después de esto quedó completado el cuestionario en su versión definitiva. Los ítems fueron calificados por los estudiantes en una escala Likert de cinco puntos: 1 = Nunca, 2 = Algunas veces, $3=$ Indeciso, $4=$ Frecuentemente y $5=$ Siempre.

Los puntajes totales de los enfoques Aprendizaje Profundo (AP) y Aprendizaje Superficial (AS) fueron construidos sumando las sub-escalas MP y EP, en el primer caso, y sumando las subescalas MS y ES, en el segundo. Puesto que todos los ítems fueron puntuados en la misma dirección, el rango de puntuaciones para el total de los ítems que componen el cuestionario osciló entre 20, como puntaje mínimo, y 100 como máximo. Asimismo, los rangos de cualesquiera subescalas de motivos y estrategias oscilaron entre 5 y 25 puntos, y el de cada una de las escalas osciló entre 10 y 50 puntos. Por ende, el enfoque de aprendizaje adoptado por el estudiante se definió por el enfoque que obtuvo la puntuación más alta. Los estudiantes que puntuaron exactamente igual en las dos escalas, combinando uno y otro enfoque, se consideró que adoptan un enfoque equilibrado (Kember, 2000).

\section{Procedimiento}

De los once grupos existentes en la carrera de psicología por cada uno de los semestres (período escolar 2019-2) se seleccionaron, aleatoriamente, dos grupos de cada uno de los semestres a examinar (segundo, cuarto y sexto). Ya realizada la selección, se habló con los profesores de cada uno de los grupos para solicitar su permiso, en aras de aplicar el cuestionario en su hora de clase. Asimismo, se les informó del propósito de la investigación y se les entregó uno de los cuestionarios para que vieran su contenido. Los profesores ofrecieron un día y hora para pasar por 
sus grupos. En cada uno de los grupos se habló con los estudiantes y se les pidió su apoyo. Además, se les informó acerca del propósito del estudio y se les aclaró que no requerían suministrar datos personales; que la información recabada sería utilizada únicamente con fines estadísticos y se aclararon las dudas sobre el contenido del cuestionario. Asimismo, se les indicó de forma clara que su participación era voluntaria. Solamente se aplicó el cuestionario a los estudiantes que aceptaron participar en el estudio.

En los estudios que aquí se reportan, como ya se mencionó, se hizo uso de los cuestionarios CHAEA de Alonso et al. (1994) y R-SPQ-2F de Biggs et al. (2001). Ambos cuestionarios fueron presentados en un cuadernillo en el cual la página inicial, la misma para todos los participantes, recababa sus datos (edad, sexo, horas dedicadas al estudio, entre otros). Las dos secciones siguientes tuvieron dos órdenes diferentes. A la mitad de la muestra se le aplicó la Versión 1, cuya secuencia fue CHAEA/R-SPQ-2, mientras que la otra mitad se le aplicó la Versión 2, cuya secuencia fue R-SPQ-2F/CHAEA. El cuadernillo con los dos cuestionarios fue contestado por los participantes en 50 minutos, aproximadamente.

\section{Análisis de datos}

Se utilizó el Paquete Estadístico para Ciencias Sociales (SPSS, 20 para Windows) para analizar los datos. Se realizaron estadísticas descriptivas para examinar las distribuciones de los cuestionarios CHAEA y R-SPQ-2, obteniendo la media, la desviación estándar y los límites inferior y superior, tanto de las escalas totales como de las subescalas (Activo Reflexivo, Teórico, Pragmático, Motivación Profunda, Estrategia Profunda, Motivación Superficial y Estrategia Superficial). Asimismo, se aplicaron pruebas de Kolmorov-Smirnov para una muestra, con el fin de observar si los puntajes se distribuían de manera normal. La prueba Alpha de Cronbach fue utilizada para determinar el nivel de confiabilidad de las escalas completas y de cada una de las subescalas. Para examinar las relaciones entre las distintas subescalas que componen los cuestionarios se utilizó una prueba ANOVA de un factor de medidas repetidas con el ajuste de Bonferroni para determinar el valor de cada una de las comparaciones. Y, con el propósito de saber si había diferencias en las respuestas de los participantes, dadas en cada una de las subescalas, respecto de las variables de contraste (semestre y promedio), se utilizó una prueba ANOVA de 3 
x 2. Por último, una prueba de correlación de Pearson examinó el grado de relación entre las cuatro subescalas que componen cada uno de los cuestionarios, así como las relaciones entre las ocho subescalas.

\section{Resultados}

\section{Cuestionario Honey-Alonso de Estilos de Aprendizaje (CHAEA)}

Se realizó un análisis para explorar si hubo efectos en la secuencia de presentación. Una prueba $t$ para muestras independientes no mostró diferencias entre las respuestas a los cuestionarios por efecto del orden de presentación, sea en la Versión 1 ( $n=111, M=217.87, D E=14.15)$ o en la Versión $2(n=110, M=216.16, D E=16.05), t(219)=.840, p>.05, d$ de Cohen $=0.11$. Las respuestas al CHAEA, tanto en la Versión 1 como en la Versión 2, fueron muy similares. Así, se realizó un análisis de cada uno de los cuestionarios por separado.

Se realizaron análisis estadísticos descriptivos con el propósito de caracterizar la muestra y los ítems que componen el cuestionario CHAEA. La exploración inicial de los datos no detectó valores perdidos, pero sí dos casos atípicos. Se analizó cada uno de los ítems a partir de sus propiedades psicométricas: media, desviación estándar, asimetría y curtosis. La confiabilidad se estimó con la prueba Alfa de Cronbach y examinando la correlación ítem-total de cada ítem, su correlación con el puntaje total y su permanencia o eliminación de la escala. Asimismo, el análisis comenzó por obtener un puntaje total de los ítems que componen cada una de las sub-escalas, con el fin de poder examinar su distribución. Por último, una prueba de normalidad de KolmogorovSmirnov para una muestra indicó que la suma total de los 80 ítems posee una distribución normal, $Z=1.107, p>.05$ (bilateral). Por su parte, un análisis del coeficiente de confiabilidad del cuestionario, a través del Alfa de Cronbach, indicó un $\alpha=.840$, con una media de los elementos de 2.79 (mínimo $=1.73$, máximo $=3.37)$ y una varianza de .583 .

Por otro lado, se procedió a examinar los índices de consistencia de cada uno de los cuatro estilos de aprendizaje que constituyen el cuestionario (20 ítems en cada uno de ellos). La Tabla 1 muestra que las propiedades psicométricas y los índices de fiabilidad en cada una de las cuatro sub-escalas son psicométricamente aceptables. Asimismo, una prueba de normalidad de 
Kolmogorov-Smirnov para una muestra encontró que dos de las cuatro sub-escalas tienen una distribución normal, pero las otras dos no se distribuyen normalmente.

\section{Tabla 1}

Propiedades psicométricas de las sub-escalas que componen el CHAEA.

\begin{tabular}{|c|c|c|c|c|c|c|c|}
\hline \multirow{2}{*}{$\begin{array}{c}\text { Sub- } \\
\text { escala }\end{array}$} & \multirow{2}{*}{$M$} & \multirow{2}{*}{$D E$} & \multicolumn{2}{|c|}{ IC 95\% } & \multirow[b]{2}{*}{$K-S$} & \multirow[b]{2}{*}{$p$} & \multirow{2}{*}{$\alpha$} \\
\hline & & & LI & $\mathbf{L S}$ & & & \\
\hline ACT & 2.69 & .312 & 2.6538 & 2.7367 & .059 & .060 & .739 \\
\hline REF & 2.97 & .313 & 2.9378 & 3.0210 & .058 & .073 & .793 \\
\hline TEO & 2.76 & .337 & 2.7189 & 2.8083 & .086 & .000 & .800 \\
\hline PARA & 2.74 & .309 & 2.7074 & 2.7894 & .087 & .000 & .733 \\
\hline
\end{tabular}

Nota: Elaboración de los autores IC = Intervalo de confianza; LI = Límite Inferior; LS = Límite Superior; ACT = Activo; REF = Reflexivo; TEO = Teórico; PRA = Pragmático.

Con el fin de examinar si existían diferencias en la elección de los estilos de aprendizaje por parte de los participantes, se realizó una prueba ANOVA de un factor con medidas repetidas. Dicha prueba mostró la existencia de diferencias estadísticamente significativas entre las medias de los cuatro estilos (véase Tabla 2). No se encontró esfericidad ( $W=.492, p<.001$ ), por lo que se reporta el criterio de Geenhouse-Geisser, $F(2.071,455.620)=45.836, p<.001$ (potencia observada $=1 ; \eta^{2}$ parcial $=.177$ ). Como se ve en la Tabla 2, comparaciones múltiples con el ajuste de Bonferroni encontraron diferencias entre ACT-REF y entre REF-TEO y REF-PRA, pero no entre ACT-TEO, ACT-PRA y TEO-PARA. Siendo que los participantes se decantan por el estilo Reflexivo y eligen en último lugar el estilo Activo. 
Tabla 2

Comparaciones múltiples con el ajuste de Bonferroni de los cuatro estilos de aprendizaje.

\begin{tabular}{|c|c|c|c|c|c|c|}
\hline \multirow[b]{2}{*}{ (I)Estilo } & \multirow[b]{2}{*}{ (J)Estilo } & \multirow{2}{*}{$\begin{array}{c}\text { Diferencia } \\
\text { de medias } \\
\text { (I-J) }\end{array}$} & \multirow{2}{*}{$\begin{array}{l}\text { Error } \\
\text { típico }\end{array}$} & \multirow[b]{2}{*}{ Sig. } & \multicolumn{2}{|c|}{ IC $95 \%$} \\
\hline & & & & & $\mathbf{L I}$ & LS \\
\hline \multirow[t]{3}{*}{$\mathrm{ACT}$} & REF & $-.284^{*}$ & .031 & .000 & -.367 & -.201 \\
\hline & TEO & -.068 & .033 & .251 & -.157 & .021 \\
\hline & PRA & -.053 & .024 & .174 & -.118 & .011 \\
\hline \multirow[t]{2}{*}{ REF } & TEO & $216^{*}$ & .018 & .000 & .168 & .263 \\
\hline & PRA & $.231^{*}$ & .025 & .000 & .165 & 297 \\
\hline TEO & PRA & .015 & .022 & 1.000 & -.044 & .074 \\
\hline
\end{tabular}

Nota: Elaboración de los autores. IC = Intervalo de confianza; LI = Límite Inferior; LS = Límite Superior; ACT = Activo; REF = Reflexivo; TEO = Teórico; PRA = Pragmático.

Una correlación de Pearson mostró, asimismo, que existen correlaciones entre los distintos estilos de aprendizaje. Como puede verse en la Tabla 3, los estilos de aprendizaje Reflexivo y Teórico mantienen una relación positiva muy fuerte, mientras que ambos mantienen relaciones negativas con el estilo de aprendizaje Activo. Ello podría suponer que la vinculación de los estilos de aprendizaje Activo-Reflexivo y Activo-Teórico son incompatibles. Por su parte, el estilo Pragmático mantiene relaciones positivas con los otros tres estilos.

\section{Tabla 3}

Índices de correlación entre los estilos de aprendizaje.

\begin{tabular}{|c|c|c|c|c|}
\hline & 1 & 2 & 3 & 4 \\
\hline 1. ACT & & -.101 & $-.165^{*}$ & $.331^{* *}$ \\
\hline 2. REF & & & $.668 * *$ & $.294 * *$ \\
\hline 3. TEO & & & 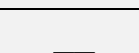 & $.478 * *$ \\
\hline
\end{tabular}




\section{PRA}

Nota: Elaboración de los autores. ACT = Activo; REF = Reflexivo; TEO = Teórico; PRA = Pragmático.

Asimismo, se examinó el efecto de las variables semestre, sistema y promedio en la elección de los cuatro estilos de aprendizaje. Una prueba ANOVA de 3 (semestre) x 2 (promedio) con los cuatro estilos como variable dependiente, no encontró efectos en ninguna de las dos variables respecto de la elección del estilo de aprendizaje $(p>.05)$.

\section{Study Processs Questionnaire revisado de dos factores (R-SPQ-2F)}

Primero se sumaron los 20 ítems que componen el cuestionario con el objetivo de obtener una puntuación total y examinar su distribución. Los resultados obtenidos mostraron que los datos se distribuyeron con una media bastante centrada en la mitad del rango de valores, de un puntaje mínimo de 27.00 a uno máximo de $75.00\left(M_{\text {Total }}=54.75, D E_{\text {Total }}=7.50\right.$, IC al 95\% [53.67, 55.82]). La prueba de normalidad de Kolmogorov-Smirnov, indicó que los datos poseen una distribución normal, $Z=.681, p>.05$.

Una prueba de confiabilidad mediante el Alfa de Cronbach indicó, para el conjunto de los 20 ítems, $\alpha=.781$. Posteriormente, se extrajeron los coeficientes de confiabilidad de las dos escalas. Así, la escala Aprendizaje Profundo obtuvo un $\alpha=.791$, con correlaciones ítem-total oscilando entre .355 y .595, mientras que la escala Aprendizaje Superficial obtuvo un $\alpha=.687$, con correlaciones ítem-total, oscilando entre .208 y .470. Asimismo, una prueba de KolmogorovSmirnov para una muestra indicó que las respuestas dadas en dos escalas: Aprendizaje Profundo y Aprendizaje Superficial, se distribuyen normalmente, $Z=.918, p>.05$ y $Z=1.316, p>.05$, respectivamente. Por otra parte, una prueba $t$ para muestras relacionadas indicó que existen diferencias estadísticamente significativas entre las medias de Aprendizaje Profundo $(M=3.48$, $D E=.650)$ y Aprendizaje Superficial $(M=1.98, D E=.516), t(220)=24.603, p<.001, d$ de Cohen $=2.55$. Una correlación de Pearson indicó que ambas escalas correlacionan negativamente, $r=-$ $.258, p<.01$.

Con el fin de examinar si existían diferencias en la aproximación al aprendizaje por parte de los participantes, se realizó una prueba ANOVA de un factor con medidas repetidas. Dicha 
prueba mostró la existencia de diferencias estadísticamente significativas entre las medias de las cuatro sub-escalas (véase Tabla 4). No se encontró esfericidad ( $W=.391, p<.001$ ), por lo que se reporta el criterio de Geenhouse-Geisser, $F(1.835,403.794)=435.623, p<.001$ (potencia observada $=1 ; \eta^{2}$ parcial $\left.=.664\right)$. Comparaciones múltiples con el ajuste de Bonferroni encontraron diferencia entre todos los pares comparados. Una comparación intra-escala (véase Tabla 5) muestra diferencias estadísticamente significativas entre las cuatro sub-escalas. En otras palabras, y dado que en Aprendizaje Profundo los participantes tienden más hacia Motivación que hacia Estrategia, mientras que en Aprendizaje Superficial esa relación se invierte (véase Tabla 4), esto quizás indica que la aproximación profunda de los estudiantes está guiada por la motivación, mientras que la aproximación superficial está guiada por la estrategia.

\section{Tabla 4}

Comparaciones múltiples con el ajuste de Bonferroni de las cuatro aproximaciones al aprendizaje

\begin{tabular}{ccccccc}
\hline \multirow{2}{*}{$(\mathbf{I})$ Subescala } & \multirow{2}{*}{$(\mathbf{J})$ Subescala } & $\begin{array}{c}\text { Diferencia de } \\
\text { medias (I-J) }\end{array}$ & $\begin{array}{l}\text { Error } \\
\text { típico }\end{array}$ & \multirow{2}{*}{ Sig. } & \multicolumn{2}{c}{ IC 95 \% } \\
\cline { 5 - 7 } & & & & & & LS \\
\hline $\mathrm{MP}$ & $\mathrm{EP}$ & $.243^{*}$ & .041 & .000 & .133 & .352 \\
\hline & $\mathrm{MS}$ & $1.701^{*}$ & .067 & .000 & 1.523 & 1.880 \\
\hline & $\mathrm{ES}$ & $1.547^{*}$ & .066 & .000 & 1.371 & 1.722 \\
\hline & & & & & & \\
\hline $\mathrm{EP}$ & $\mathrm{MS}$ & $1.459^{*}$ & .066 & .000 & 1.283 & 1.634 \\
\hline & $\mathrm{ES}$ & $1.304^{*}$ & .069 & .000 & 1.120 & 1.489 \\
\hline & & & & & & \\
\hline $\mathrm{MS}$ & $\mathrm{ES}$ & $-.155^{*}$ & .037 & .000 & -.254 & -.055 \\
\hline
\end{tabular}

Nota: Elaboración de los autores. IC = Intervalo de confianza; LI = Límite Inferior; LS = Límite Superior; MP = Motivación Profunda; EP = Estrategia Profunda; MS = Motivación Superficial; $\mathrm{ES}=$ Estrategia Superficial.

Como puede verse en los datos obtenidos, los participantes se decantan más por el enfoque de aprendizaje Profundo que por el Superficial; sin embargo, de acuerdo con las medias obtenidas, en Aprendizaje Profundo los participantes tienden más hacia la sub-escala Motivación Profunda $(M=3.60, D E=.716)$ que hacia la sub-escala Estrategia Profunda $(M=3.36, D E=.720)$; mientras 


\section{Enfoques y estilos \\ de aprendizaje}

Enfoques y estilo de aprendizaje | Revista Virtual

Universidad Católica del Norte, 65, 102-136

ISSN: 0124-5821 (En línea)

que en Aprendizaje Superficial invierten esa relación: Motivación Superficial $(M=1.90, D E=$ $.556)$ y Estrategia Superficial $(M=2.05, D E=.615$.

Una prueba de Pearson (Tabla 5) muestra que las sub-escalas de Aprendizaje Profundo (MP y EP) correlacionan fuerte y positivamente al igual que las sub-escalas de Aprendizaje Superficial (MS y ES), mientras que las relaciones Profundo y Superficial son negativas, tal y como se esperaría.

\section{Tabla 5}

Índices de correlación entre los enfoques de aprendizaje

\begin{tabular}{|c|c|c|c|c|}
\hline & 1 & 2 & 3 & 4 \\
\hline 1. MP & -- & $.637 * *$ & $-.215^{*}$ & -.079 \\
\hline 2. EP & & - & $-.165^{*}$ & $-.183^{*}$ \\
\hline 3. MS & & & -- & $.554 * *$ \\
\hline
\end{tabular}

4. ES

Nota: Elaboración de los autores. MP = Motivación Profunda; $\mathrm{EP}=$ Estrategia Profunda; $\mathrm{MS}=$ Motivación Superficial; ES = Estrategia Superficial.

Una prueba ANOVA de 3 (semestre) x 2 (promedio), con las cuatro sub-escalas como variable dependiente, no encontró efectos de ninguna de las dos variables respecto de la elección del estilo de aprendizaje $(p>.05)$.

Por último, una prueba de Pearson mostró relaciones fuertes y positivas entre los estilos de aprendizaje REF, TEO y PRA y las sub-escalas MP y EP. Y una relación débil y positiva entre ACT y MP, pero sin relación entre ACT y EP (Tabla 6). Es decir, la dimensión Aprendizaje Profundo del R-SPQ-2F correlaciona fuertemente con los estilos de aprendizaje del CHAEA. 


\section{Tabla 6}

Índices de correlación entre los componentes de CHAEA y R-SPQ-2F

\begin{tabular}{ccccc}
\hline & ACT & REF & TEO & PRA \\
\hline MP & & & & \\
\hline & $.166^{*}$ & $.302^{* *}$ & $.399^{* *}$ & $.302^{* *}$ \\
\hline EP & .131 & $.314^{* *}$ & $.369^{* *}$ & $.303^{* *}$ \\
\hline MS & & & & \\
\hline & -.014 & -.091 & -.043 & .079 \\
\hline ES & .047 & .002 & -.020 & .023 \\
\hline
\end{tabular}

Nota : Elaboración de los autores. ACT $=$ Activo; REF $=$ Reflexivo; TEO $=$ Teórico; PRA $=$ Pragmático; MP = Motivación Profunda; EP = Estrategia Profunda; MS = Motivación Superficial; $\mathrm{ES}=$ Estrategia Superficial .

Los datos obtenidos a partir de los dos instrumentos utilizados en el estudio (CHAEA y RSPQ-2F) muestran un perfil de respuestas; a saber, la preferencia de los estilos Reflexivo y Teórico en la elección de los participantes y una mayor inclinación hacia el enfoque Profundo. Dicha tendencia parece sintetizarse en la fuerte relación que se observa, en la tabla 6, entre ellos.

\section{Discusión}

Los hallazgos de este estudio muestran, por un lado, que el instrumento CHAEA posee muy buenas propiedades psicométricas y logra diferenciar adecuadamente entre los cuatro estilos de aprendizaje, mostrando que los participantes seleccionan, en primer lugar, el estilo Reflexivo, seguido del Teórico, Pragmático y, por último, el Activo. Y, por el otro, que los datos obtenidos se ajustan a las predicciones teóricas (Alonso et al., 1994); a saber, se encontró que las relaciones entre los estilos de aprendizaje Reflexivo y Teórico son fuertes y positivas, mientras que las relaciones de ambos con el estilo de aprendizaje Activo son negativas (incompatibles) (véase Tabla 3). En otras palabras, los datos obtenidos en este estudio corroboran dicha aserción y compaginan con los reportados por otros estudios, en los cuales el estilo de aprendizaje marcadamente encontrado es el Reflexivo (Alonso et al., 1994; Altamirano-Droguett et al., 2019; Bravo et al., 2020; Esguerra y Guerrero, 2010; Gil Madrona et al., 2007; Martínez et al., 2019; Ordoñez et al., 
2003; Ortiz y Canto, 2013), o bien, una combinación entre los estilos Reflexivo y Teórico (Camarero et al., 2000).

No obstante, si bien se ha encontrado que los estudiantes universitarios tienden hacia los estilos de aprendizaje Reflexivo y Teórico (Bahamón et al., 2012) en algunos casos, por ejemplo, estudiantes de educación a distancia tienden hacia el estilo Activo (Gómez et al., 2017), pero ello solo ocurre en los estudiantes de primer semestre, en donde predominan los estilos de aprendizaje Activo y Teórico, situación que cambia en el décimo semestre en el que predominan los estilos Reflexivo y Pragmático. Otros investigadores (Freiberg-Hoffmann et al., 2017) han encontrado que estudiantes de psicología, física e ingeniería industrial, de primer ingreso, tienden hacia el estilo Pragmático. Por el otro lado, si bien los participantes se decantan por el estilo de aprendizaje Reflexivo, de manera similar a lo encontrado en otras investigaciones realizadas con estudiantes universitarios (Bahamón et al., 2012; Camarero et al., 2000; Gil Madrona et al., 2007; Ortiz y Canto, 2013), llama la atención que ninguna de las variables que se utilizaron para contrastar las respuestas de los participantes, a saber: "promedio" y "semestre", incidiera en la elección de un determinado estilo de aprendizaje.

Respecto de los enfoques de aprendizaje, los datos obtenidos en el presente estudio indican que los participantes tienden hacia el enfoque Profundo, lo cual concuerda con lo hallado en otros estudios (Astika \& Sumakul, 2020; Bana \& Rizvi, 2019; Díaz-García et al., 2020; Hernández Pina et al., 2010; Mansfield et al., 2020). Algunos autores, como Kember \& Gow (1989), han encontrado que estudiantes con una marcada predisposición hacia un enfoque Profundo llegan a emplear estrategias de aprendizaje "superficiales", simplemente porque la tarea de aprendizaje parece exigirlo así. Sin embargo, aunque la tarea exija el uso de estrategias "profundas”, los estudiantes con una aproximación superficial hacia el aprendizaje no llegan a utilizarlas, algo que sí hacen los estudiantes con un enfoque Profundo (Díaz-García et al., 2020).

Esto parecería estar relacionado con los datos aportados por otras investigaciones. Por ejemplo, los estudiantes que emplean con mayor frecuencia y eficacia estrategias de autorregulación son aquellos que poseen un enfoque Profundo (Rosário, Núñez, González-Pienda, Valle et al., 2010). Todos estos datos quizá fortalezcan la idea de que aproximarse de una forma u otra al aprendizaje depende de la percepción que tengan los estudiantes del contenido, contexto y demandas particulares de las tareas que se les presentan (Alonso et al., 1994; Bana \& Rizvi, 2019; 
Díaz-García et al., 2020). Por ejemplo, Freiberg-Hoffmann, Berenguer et al. (2017) encontraron que los estudiantes de primer ingreso a la universidad tienden a utilizar el enfoque Superficial, ponderando la utilización de estrategias memorísticas y repetitivas para utilizar la información. Con todo, queda por resolver por qué estudiantes con las mismas percepciones adoptan diferentes aproximaciones (véase Richardson, 2010).

En suma, los resultados obtenidos en este estudio son similares a los encontrados en otras investigaciones, sea que hayan utilizado el CHAEA o el R-SPQ-2F; a saber, los estudiantes universitarios tienden hacia los estilos Reflexivo y Teórico, por un lado, y hacia el enfoque Profundo, por el otro. Este hecho quizás indica que las actividades que se desarrollan en la educación universitaria requieren y fomentan ciertas estrategias y formas de procesar la información, lo que influye y guía los estilos y enfoques de aprendizaje de los estudiantes. Sin embargo, no debe descartarse que la propia cultura académica puede hacer lo mismo desde los niveles escolares básicos. Ello explicaría, en todo caso, porqué algunos estudiantes que aplican a la carrera de medicina, y los que ya la cursan, se decanten por el enfoque Profundo (Mansfield et al., 2020).

Asimismo, la literatura aquí revisada muestra que el CHAEA o el R-SPQ-2F son instrumentos confiables para examinar los estilos y los enfoques de aprendizaje, respectivamente. No obstante, pocos trabajos han utilizado ambos en un afán por constatar la dinámica de los dos constructos. En latinoamérica se tiene el caso de Freiberg-Hoffmann, Berenguer et al. (2017), pero, hasta donde sabemos, un trabajo similar no se ha realizado en México. Por esa razón, en este estudio se decidió utilizar los dos cuestionarios, en tanto se cree que examinar solo los enfoques de aprendizaje o los estilos de aprendizaje no aporta evidencia clara de por qué los estudiantes optan por una u otra opción. Examinar las respuestas a ambos instrumentos, de forma conjunta, ilumina de mejor manera cómo se relacionan ambos constructos.

\section{Conclusión}

En el presente estudio, si bien los participantes tendieron a ponderar en mayor medida el estilo Reflexivo, lo cierto es que el porcentaje de elección del estilo Activo (ubicado en último lugar) no fue menor; fue elegido el $67.25 \%$ de las veces. Es decir, el predominio de un estilo de 
aprendizaje no nulifica la presencia de los otros, incluso aunque alguno de ellos sea incompatible con algún otro; por ejemplo, Reflexivo versus Activo.

Los datos obtenidos también indican que las relaciones entre el CHAEA y el R-SPQ-2F se dan como cabría esperar. Es decir, el CHAEA no indica que un determinado estilo de aprendizaje deba de prevalecer sobre otro, como si estuvieran organizados de acuerdo con una jerarquía creciente, lo cual si ocurre con el R-SPQ-2F. En tal caso, es coherente que el enfoque Profundo correlacione con los cuatro estilos de aprendizaje, pero no lo haga el enfoque Superficial (véase Tabla 6). Esto quizá quiera decir que el enfoque de aprendizaje es crucial para determinar el perfil del estudiante y que, con independencia de que apruebe o no sus asignaturas, esto lo hará de dos modos distintos y con cualidad diferente; a saber, tenderá a memorizar los contenidos o a comprenderlos. Sin embargo, ello no ocurre con los estilos de aprendizaje, los cuales parecen ser más bien una adecuación a las distintas tareas y requisitos académicos que el estudiante tiene que afrontar en su recorrido académico.

Con todo, es claro que desde la práctica educativa se necesitan conocer tanto los estilos como los enfoques de los estudiantes, con el fin de organizar y enfocar la enseñanza hacia los mismos, y de esa manera incidir en su rendimiento académico. Pero, más importante aún es el hecho de potenciar aquellos aspectos que mejoren su trabajo académico; por ejemplo, a través de fomentar el enfoque Profundo, en tanto se sabe que los estudiantes que sostienen este enfoque son más autónomos, más persistentes y tienen un mejor rendimiento académico que quienes sostienen un enfoque Superficial, o bien, que disponen de mejores mecanismos para enfrentar la ansiedad. En este tenor, el trabajo aquí presentado invita a utilizar el CHAEA y el R-SPQ-2F como herramientas válidas y confiables para diagnosticar los estilos y los enfoques de aprendizaje de los estudiantes con anterioridad a su inserción en el aula. Un diagnóstico que daría a conocer los perfiles necesarios para que el profesorado organice, diseñe y gradué las actividades de enseñanzaaprendizaje.

Por último, asumiendo que la práctica educativa se organiza en torno de los componentes de la agencia académica, y que las creencias epistemológicas son omnipresentes a lo largo de todo el proceso, parece necesario que futuras indagatorias aborden los vínculos entre las creencias epistemológicas y los enfoques de aprendizaje, y que al hacerlo examinen las posibles etapas que sigue un estudiante a lo largo del proceso. Necesario, porque se sabe que a medida que los 
estudiantes avanzan en sus estudios, cambian tanto sus creencias epistemológicas (se hacen más sofisticadas) como sus enfoques de aprendizaje (tienden más a lo profundo); y, además, porque las creencias epistemológicas predicen significativamente el rendimiento académico, tanto directa como indirectamente, a través de los enfoques de aprendizaje. De la misma manera, si bien en los estudiantes de primer semestre de educación universitaria predominan los estilos de aprendizaje Activo y Teórico, dicha tendencia cambia en el décimo semestre en el que predominan los estilos Reflexivo y Pragmático. De la misma manera, los estudiantes de primer ingreso a la universidad tienden a utilizar el enfoque Superficial, ponderando la utilización de estrategias memorísticas y repetitivas para utilizar la información y después hacer un cambio hacia el enfoque Profundo.

Quizá de esta manera, de forma tangencial, también se podría saber un poco más acerca de los estilos de aprendizaje vinculando este constructo con aspectos sustanciales de la esfera motivacional. Por ejemplo, se sabe que los estudiantes con creencias teóricamente sofisticadas, que creen que la fuente de conocimiento es interna en lugar de externa, tienen más probabilidades de valorar el aprendizaje Profundo y el dominio de tareas, adoptando así metas de dominio. Por el contrario, los estudiantes que creen en autoridades omniscientes tienen más probabilidades de tener un enfoque externo y, por tanto, adoptar metas de desempeño. Todo ello iluminaría de mejor manera un proceso que se desempeña en un contexto de interrelaciones y normas sociales, pero que son moduladas por la agencia de los estudiantes.

\section{Referencias}

Abalde, E., Barca, A., Muñoz, J. M., y Ziemer, M. (2009). Rendimiento académico y enfoques de aprendizaje: Una aproximación a la realidad de la enseñanza superior brasileña en la región norte. Revista de Investigación Educativa, 27(2), 303-319. http://www.redalyc.org/articulo.oa?id=283321906002

Adán, M. I. (2008). Los estilos de aprendizaje en la orientación y tutoría de bachillerato. Revista $\begin{array}{llll}\text { Complutense } & \text { de } & \text { Educación, } & \text { 19(1), }\end{array}$ https://revistas.ucm.es/index.php/RCED/article/view/RCED0808120059A

Alexander, P., Schallert, D., \& Reynolds, R. (2009). What is learning anyway? A topographical perspective considered $[¿$ Qué es aprender de todos modos? Una perspectiva topográfica 
Enfoques y estilo de aprendizaje | Revista Virtual Universidad Católica del Norte, 65, 102-136 ISSN: 0124-5821 (En línea)

considerada]. Educational
Psychologist,

44(3),

$176-192$.

Alonso, C., Gallego, D., y Honey, P. (1994). Los estilos de aprendizaje: Procedimientos de diagnóstico y mejora. Ediciones Mensajero.

Alonso, C., y Gallego, D. (2010). Los estilos de aprendizaje como competencias para el estudio, el trabajo y la vida. Revista Estilos de Aprendizaje, 6(3), 4-22. http://revistaestilosdeaprendizaje.com/article/view/909

Altamirano-Droguett, J. E., Araya-Crisóstomo, S. P., y Paz, M. (2019). Estilos de aprendizaje y rendimiento académico de estudiantes de la carrera de obstetricia. Revista Ciencias de la Salud, 17(2), 276-292. http://dx.doi.org/10.12804/

Arenas, E. P. (2017). Estrategias de estilos de aprendizaje de estudiantes: Proceso de validación 1. ALTERIDAD. Revista de Educación, 12(2), 224-237. https://doi.org/10.17163/alt.v12n2. 2017.08

Astika, G., \& Sumakul, D. T. (2020). Students'profiles through learning approaches using Biggs'Study Process Questionnaire [Perfiles de los estudiantes a través de enfoques de aprendizaje mediante el Cuestionario de proceso de estudio de Biggs]. English Language Teaching and Research Journal, 3(1), 46-54. https://doi.org/10.37147/eltr.2019.030104

Bahamón, M., Vianchá, M., Alarcón, L., y Bohórquez, C. (2012). Estilos y estrategias de aprendizaje: una revisión empírica y conceptual de los últimos años. Pensamiento Psicológico, 10(1), 129-144. https://www.redalyc.org/pdf/801/80124028009.pdf

Bana, K. F. M. A., \& Rizvi, K. F. (2019). Comparing the learning approaches using Biggs Revised Study Process Questionnaire (R-SPQ-2F) among dental undergraduates [Comparación de los enfoques de aprendizaje mediante el Cuestionario de proceso de estudio revisado de Biggs (R-SPQ-2F) entre estudiantes de odontología]. Journal of the Pakistan Dental Association, 28(02), 68-73. https://doi.org/10.25301/JPDA.282.68

Biggs, J. (1987). Study Process Questionnaire Manual. Student approaches to learning and studying [Manual del cuestionario del proceso de estudio. Enfoques de los estudiantes para aprender y estudiar]. Education Resources Information Center (ERIC). http://www.eric.ed.gov/PDFS/ED308200.pdf 
Biggs, J., Kember, D., \& Leung, D. Y. P. (2001). The revised two-factor study process questionnaire: R-SPQ-2F. British Journal of Educational Psychology, 71(1), 133-149. https://doi.org/10.1348/000709901158433

Bravo, P. N., Schubert, V. M., y Pedroso, B. (2020). Estilos de aprendizaje: Preferencia de los estudiantes de enfermería de la Universidad de Magallanes, Chile. Texto \& ContextoEnfermagem, 29(spe), 1-12. https://doi.org/10.1590/1980-265X-TCE-2019-0265

Brownlee, J. L., Ferguson, L. E., \& Ryan, M. (2017). Changing teachers' epistemic cognition: A new conceptual framework for epistemic reflexivity [Cambiando la cognición epistémica de los profesores: un nuevo marco conceptual para la reflexividad epistémica]. Educational Psychologist, 52(4), 242-252. https://doi.org/10.1080/00461520.2017.1333430

Buendía, L., y Olmedo, E. (2003). Estudio transcultural de los enfoques de aprendizaje en educación superior. Revista de Investigación Educativa, 21(2), 371-386. https://revistas.um.es/rie/article/view/99261

Camarero, F., del Buey, F., y Herrero, J. (2000). Estilos y estrategias de aprendizaje en estudiantes universitarios. Psicothema, 12(4), 615-622. http://www.psicothema.es/pdf/380.pdf

Cassidy, S. (2004). Learning styles: an overview of theories, models, and measures [Estilos de aprendizaje: una descripción general de teorías, modelos y medidas]. Educational Psychology, 24(4), 419-444. https://www.tandfonline.com/doi/full/10.1080/0144341042000228834

Castañeda, S. (2004). Evaluando y fomentando el desarrollo cognitivo y el aprendizaje complejo. $\begin{array}{lllll}\text { Psicología desde el } & \text { Caribe, } & 13(1), & \text { 109-143. }\end{array}$ https://www.redalyc.org/pdf/213/21301307.pdf

Castañeda, S., y Ortíz, B. D. (2017). El rol de las creencias epistemológicas en el aprendizaje académico en psicología. En Z. Monroy, R. León-Sánchez y G. Álvarez (Coords.), Obstáculos epistemológicos en la enseñanza y el aprendizaje de la filosofía y de la ciencia (pp. 263-282). Universidad Nacional Autónoma de México.

Chan, K. W. (2003). Hong Kong teacher education students' epistemological beliefs and approaches to learning [Creencias epistemológicas y enfoques del aprendizaje de los estudiantes de formación docente de Hong Kong]. Research in Education, 69(1), 36-50. https://doi.org/10.7227/RIE.69.4 
Cimermanová, I. (2018). The effect of learning styles on academic achievement in different forms of teaching [El efecto de los estilos de aprendizaje en el rendimiento académico en diferentes formas de enseñanza]. International Journal of Instruction, 11(3), 219-232. https://eric.ed.gov/?id=EJ1183439

Cornejo, R., y Redondo, J. M. (2007). Variables y factores asociados al aprendizaje escolar. Una discusión desde la investigación actual. Estudios Pedagógicos, 33(2), 155-175. http://dx.doi.org/10.4067/S0718-07052007000200009

Cuthbert, P. (2005). The student learning process: learning styles or learning approaches? [El proceso de aprendizaje del estudiante: ¿estilos de aprendizaje o enfoques de aprendizaje?] $\begin{array}{llll}\text { Teaching in } \quad \text { Higher } & \text { 235-249. }\end{array}$ https://doi.org/10.1080/1356251042000337972

Dantas, L. A., \& Cunha, A. (2020). An integrative debate on learning styles and the learning process [Un debate integrador sobre los estilos de aprendizaje y el proceso de aprendizaje]. Social Sciences \& Humanities Open, 2(1), Artículo 100017. https://doi.org/10.1016/j.ssaho.2020.100017

Demirbas, O., \& Demirkan, H. (2007). Learning styles of design and the relationship of academic performance and gender in design education [Estilos de aprendizaje de diseño y la relación del rendimiento académico y el género en la educación del diseño]. Learning and Instruction, 17(3), 345-359. https://doi.org/10.1016/j.learninstruc.2007.02.007

De Vita, G. (2001). Learning styles, culture and inclusive instruction in the multicultural classroom: A business and management perspective [Estilos de aprendizaje, cultura e instrucción inclusiva en el aula multicultural: una perspectiva empresarial y de gestión]. Innovations in Education and Teaching International, 38(2), 165-174. https://doi.org/10.1080/14703290110035437

Díaz-García, I., Almerich, G., Suárez-Rodríguez, J., y Orellana, N. (2020). La relación entre las competencias TIC, el uso de las TIC y los enfoques de aprendizaje en alumnado universitario de educación. Revista de Investigación Educativa, 38(2), 549-566. http://dx.doi.org/10.6018/rie.409371

Díaz-Véliz, G., Mora, S., Lafuente-Sánchez, J., Gargiulo, P., Bianchi, R., Terán, C., Gorena, D., Arce, J., y Escanero-Marcen J. (2009). Estilos de aprendizaje de estudiantes de medicina 
en universidades latinoamericanas y españolas: relación con los contextos geográficos y $\begin{array}{llll}\text { curriculares. } & \text { Educación } & \text { Médica, } & \text { 12(3), }\end{array}$ https://scielo.isciii.es/scielo.php?script=sci_arttext\&pid=S1575-18132009000400008

Duff, A., \& Duffy, T. (2002). Psychometric propierties of Honey \& Mumford's Learning Styles Questionnaire (LSQ) [Propiedades psicométricas del cuestionario de estilo de aprendizaje de Honey \& Mumford (LSQ)]. Personality and Individual Differences, 33(1), 147-163. https://doi.org/10.1016/S0191-8869(01)00141-6

Duff, A., \& McKinstry, S. (2007). Students' approaches to learning [Enfoques de aprendizaje de los estudiantes]. Issues in Accounting Education, 22(2), 183-214. https://doi.org/10.2308/iace.2007.22.2.183

Dunn, R. (1984). Learning style: state of the science [Estilo de aprendizaje: estado de la ciencia]. Theory into Practice, 23(1), 10-19. https://doi.org/10.1080/00405848409543084

Entwistle, N. J. (1991). Approaches to learning and perceptions of the learning environment. Introduction to the special issue [Enfoques del aprendizaje y percepciones del entorno de aprendizaje. Introducción al número especial]. Higher Education, 22, 201-204. https://link.springer.com/article/10.1007/BF00132287

Esguerra, G., y Guerrero, P. (2010). Estilos de aprendizaje y rendimiento académico en estudiantes de Psicología. Diversitas: Perspectivas en Psicología, 6(1), 97-109. https://www.redalyc.org/articulo.oa?id=67916261008

Esquivel, J., Rodríguez, M. C., y Padilla, V. M. (2009). Enfoques hacia el aprendizaje, motivos y estrategias de estudiantes de las carreras de enfermería, ingeniería y organización deportiva. Revista de Pedagogía, 30(87), $\quad 309-331$. http://www.scielo.org.co/pdf/dpp/v6n1/v6n1a08.pdf

Freiberg-Hoffmann, A., Abal, F., y Fernández-Liporace, M. (2020). Cuestionario Honey-Alonso de Estilos de Aprendizaje: Nuevas evidencias psicométricas en población argentina. Revista Colombiana de Psicología, 23(2), 328-338. https://doi.org/10.14718/acp.2020.23.2.13

Freiberg-Hoffmann, A., Berenguer, D., Fernandez-Liporace, M. M., y Ledesma, R. D. (2017). Estilos, estrategias y enfoques de aprendizaje en estudiantes universitarios de Buenos Aires. Psicodebate, 17(1), 2-34. https://doi.org/10.18682/pd.v17i1.626 
Freiberg-Hoffmann, A., y Fernández-Liporace, M. M. (2016). Enfoques de aprendizaje en estudiantes universitarios argentinos según el R-SPQ-2F: Análisis de sus propiedades psicométricas. Revista Colombiana de Psicología, 25(2), 327-329. https://doi.org/10.15446/rcp.v25n2.51874

Fryer L. K., \& Vermunt, J. D. (2018). Regulating approaches to learning: Testing learning strategy convergences across a year at university [Regular los enfoques del aprendizaje: probar las convergencias de las estrategias de aprendizaje a lo largo de un año en la Universidad]. British Journal of Educational Psychology, 88(1), 21-41. https://doi.org/10.1111/bjep.12169

García, M. R., y Sebastián, C. (2011). Creencias epistemológicas de estudiantes de pedagogía en educación parvularia, básica y media: ¿Diferencias en la formación inicial docente? Psykhe, 20(1), 29-43. http://dx.doi.org/10.4067/S0718-22282011000100003

Gil Madrona, P., Contreras, O., Pastor, J. C., Gómez, I., González, S., García, L. M., de Moya, M., y López, A. (2007). Estilos de aprendizaje de los estudiantes de magisterio: especial consideración de los alumnos de educación física. Profesorado: Revista de Curriculum y Formación del Profesorado, $\quad 11(2), \quad 1-19$. https://www.redalyc.org/articulo.oa?id=56711209

Gómez, E., Jaimes, J., y Severiche, C. (2017). Estilos de aprendizaje en universitarios, modalidad de educación a distancia. Revista Virtual Universidad Católica del Norte, (50), 383-393. http://revistavirtual.ucn.edu.co/index.php/RevistaUCN/article/view/829/1347

Hernández Pina, F., Rodríguez, Ma. C., Ruiz, E., y Esquivel, J. (2010). Enfoques de aprendizaje en alumnos universitarios de la titulación de ciencias de la actividad física y del deporte de España y México. Revista Iberoamericana de Educación, 53(7), 1-11. https://doi.org/10.35362/rie5371695

Hofer, B. K. (2004). Exploring the dimensions of personal epistemology in differing classroom contexts: Student interpretations during the first year of college [Explorando las dimensiones de la epistemología personal en diferentes contextos de aula: interpretaciones de los estudiantes durante el primer año de la Universidad]. Contemporary Educational Psychology, 29(2), 129-163. https://doi.org/10.1016/j.cedpsych.2004.01.002 
Hofer, B. K., \& Pintrich, P. R. (1997). The development of epistemological theories: Beliefs about knowledge and knowing and their relation to learning [El desarrollo de las teorías epistemológicas: creencias sobre el conocimiento y el saber y su relación con el aprendizaje]. Review of Educational Research, 67(1), 88-140. https://doi.org/10.3102/00346543067001088

Hofer, B. K., \& Sinatra, G. M. (2010). Epistemology, metacognition, and self-regulation: Musings on an emerging field [Epistemología, metacognición y autorregulación: reflexiones sobre un campo emergente]. Metacognition and Learning, 5(1), 113-120. https://link.springer.com/article/10.1007\%2Fs11409-009-9051-7

Juárez, C. S. (2014). Propiedades psicométricas del cuestionario Honey-Alonso de Estilos de Aprendizaje (CHAEA) en una muestra mexicana. Revista de Estilos de Aprendizaje, 7(13), 136-154. http://revistaestilosdeaprendizaje.com/article/view/1011

Juárez, C. S.., Rodríguez, G., Escoto, M., y Luna E. (2016). Relación de los estilos de aprendizaje y estrategias de aprendizaje con el rendimiento académico en estudiantes universitarios. $\begin{array}{lllll}\text { Journal of } & \text { Learning } & \text { Styles, } & 9(17), & \text { 268-288. }\end{array}$ http://revistaestilosdeaprendizaje.com/article/view/1054

Justicia, F., Pichardo, M. C., Cano, F., Berbén, A. B. G., \& de la Fuente, J. (2008). The revised Two-Factor Study Process Questionnaire (R-SPQ-2F): Exploratory and confirmatory factor analyses at item level [El cuestionario revisado del proceso de estudio de dos factores (R-SPQ-2F): análisis factorial exploratorio y confirmatorio a nivel de ítem]. European Journal of Psychology of Education, 23(3), 355-372. https://doi.org/10.19083/ridu.7.190

Karagiannopoulou, E., Milienos, F. S., Kamtzios, S., \& Rentzios, Ch. (2020). Do defense styles and approaches learning 'fit together in students' profiles? Differences between years of study [¿Los estilos de defensa y los enfoques de aprendizaje "encajan en los perfiles de los estudiantes?" Diferencias entre años de estudio]. Educational Psychology, 40(5), 570-591. https://doi.org/10.1080/0144 3410. 2019.1600661

Kember, D. (2000). Misconceptions about the learning approaches, motivation and study practices of Asian students [Conceptos erróneos sobre los enfoques de aprendizaje, la motivación y las prácticas de estudio de los estudiantes asiáticos]. Higher Education, 40(1), 99-121. https://doi.org/10.1023/A:1004036826490 
Kember, D., \& Gow, L. (1989). A model of student approaches to learning encompassing ways to influence and change approaches [Un modelo de enfoques de aprendizaje de los estudiantes que abarca formas de influir y cambiar los enfoques]. Instructional Science, 18(4), 263288. https://doi.org/10.1007/BF00118014

Kirschner, P. A., \& Van Merriënboer, J. J. G. (2008). Ten steps to complex learning: A new approach to instruction and instructional design [Diez pasos para el aprendizaje complejo: un nuevo enfoque para la instrucción y el diseño instruccional]. In T. L. Good (Ed.), 21st century education: A reference handbook (pp. 244-253). SAGE Publications. https://core.ac.uk/download/pdf/55535269.pdf

Kolb, A., \& Kolb, D. (2005a). Learning styles and learning spaces: Enhancing experiential learning in higher education [Estilos de aprendizaje y espacios de aprendizaje: potenciar el aprendizaje experiencial en la educación superior]. Academy of Management Learning \& Education, 4(2), 193-212. https://doi.org/10.5465/amle.2005.17268566

Kolb, A., \& Kolb, D. (2005b). The Kolb Learning Style Inventory-Version 3.1: 2005. Technical specifications [The Kolb Learning Style Inventory-Versión 3.1: 2005. Especificaciones técnicas]. http://www.whitewater-rescue.com/support/pagepics/ 1sitechmanual.pdf

Laffita, P., y Guerrero, E. (2017). Estilos de aprendizaje y autoeficacia académica. Journal of Learnign Styles, 10(17), 92-111. http://revistaestilosdeaprendizaje.com/article/view/1071

Landrum, T. J., \& McDuffie, K. (2010). Learning styles in the age of differentiated instruction [Estilos de aprendizaje en la era de la instrucción diferenciada]. Exceptionality, 18(1), 617. https://doi.org/10.1080/09362830903462441

Leiva-Brondo, M., Cebolla-Cornejo, J., Peiró, R., Andrés-Colas, N., Esteras, C., Ferriol, M., Merle, H., Díez, M. J., \& Pérez-de-Castro, A. (2020). Study approaches of life science students using the revised Two-Factor Study Process Questionnaire (R-SPQ-2F) [Estudiar los enfoques de los estudiantes de ciencias de la vida utilizando el Cuestionario revisado del proceso de estudio de dos factores (R-SPQ-2F)]. Education Sciences, 10(7), 173. https://doi.org/10.3390/educsci10070173

Lonka, K., Ketonen, E., \& Vermunt, J. D. (2021). University students' epistemic profiles, conceptions of learning, and academic performance [Perfiles epistémicos, concepciones 
del aprendizaje y desempeño académico de los estudiantes universitarios]. Higher Education, 81(4), 775-793. https://doi.org/10.1007/s10734-020-00575-6

López, M., y López, A. I. (2013). Los enfoques de aprendizaje. Revisión conceptual y de investigación. Revista Colombiana de Educación, 64, 131-153. https://www.redalyc.org/pdf/4136/413634076006.pdf

López-Aguado, M., \& Gutiérrez-Provecho, L. (2018). Checking the underlaying structure of RSPQ-2F using covariance structure analysis [Comprobación de la estructura subyacente de R-SPQ-2F mediante análisis de estructura de covarianza]. Cultura \& Educación, 30(1), 105-141. https://doi.org/10.1080/11356405.2017.1416787

López-Vargas, O., Hederich-Martínez, C., y Camargo-Uribe, A. (2011). Estilo cognitivo y logro $\begin{array}{lllll}\text { académico. } & \text { Educación } & y & \text { Educadores, } & \text { 14(1), }\end{array}$ http://www.scielo.org.co/pdf/eded/v14n1/v14n1a05.pdf

Mansfield, K. J., Peoples, G. E., Parker-Newlyn, L., \& Skropeta, D. (2020). Approaches to learning: Does medical school attract students with the motivation to go deeper? [Enfoques de aprendizaje: ¿La facultad de medicina atrae a estudiantes con la motivación de profundizar más?]. Education Sciences, 10, 302. https://doi.org/10.3390/educsci10110302

Martínez, I. M., Arellano, P. R., y Martínez, P. (2019). Los estilos de aprendizaje y de enseñanza: análisis y diagnóstico en educación superior de centro internacional de estudios superiores del español, CIESE-Comillas (España). Revista de Estilos de Aprendizaje, 12(24), 28-41. http://revistaestilosdeaprendizaje.com/article/view/1317

McLoughlin, C. (1999). The implications of the research literature on learning styles for the design of instructional material. Australasian Journal of Educational Technology, 15(3), 222-241. https://doi.org/10.14742/ajet.1859

Mohamed, M. T., \& El-Habbal, M. (2013). The relationship between epistemic beliefs and academic performance: Are better students always more mature? [La relación entre creencias epistémicas y rendimiento académico: ¿Los mejores estudiantes son siempre más maduros?]. Journal of Educational and Developmental Psychology, 3(1), 158-172. https://doi.org/10.5539/jedp.v3n1p158 
Monroy, F., y Hernández Pina, F. (2014). Factores que influyen en los enfoques de aprendizaje universitario. Una revisión sistemática. Educación XX1,17(2), 105-124. https://doi.org/10.5944/educxx1.17.2.11481

Ordoñez, F., Rosety-Rodríguez, M., y Rosety-Plaza, M. (2003). Análisis de los estilos de aprendizaje entre los estudiantes de ciencias de la salud. Enfermería Global, (3), 1-6. http://revistas.um.es/eglobal/article/view/619/645

Ortiz, F., y Canto, P. (2013). Estilos de aprendizaje y rendimiento académico en estudiantes de ingeniería en México. Revista Estilos de Aprendizaje, 11(11), 160-177. http://revistaestilosdeaprendizaje.com/article/view/978

Osher, D., Cantor, P., Berg, J., Steyer, L., \& Rose, T. (2020). Drivers of human development: How relationships and contexts shape learning and development [Factores impulsores del desarrollo humano: cómo las relaciones y los contextos moldean el aprendizaje y el desarrollo]. Applied Developmental Science, 24(1), 6-36. https://doi.org/10.1080/10888691.2017.1398650

Patiño-Toro, O. N., Bermeo-Giraldo, C., Valencia-Arias, A., y Garcés-Giraldo, L. F. (2020). Factores que inciden en el aprendizaje en gestión tecnológica e innovación en estudiantes de administración mediante el modelo de aceptación tecnológica. Formación universitaria, 13(5), 77-86. http://dx.doi.org/10.4067/S0718-50062020000500077

Paulsen, M. B., \& Feldman, K. A. (2005). The conditional and interaction effects of epistemological beliefs on the self-regulated learning of college students: Motivational strategies [Los efectos condicionales y de interacción de las creencias epistemológicas en el aprendizaje autorregulado de estudiantes universitarios: estrategias motivacionales]. Research in Higher Education, 46(7), 731-768. https://doi.org/10.1007/s11162-004-6224$\underline{8}$

Phan, H. P. (2008). Exploración de las creencias epistemológicas y los enfoques de aprendizaje en su contexto: una perspectiva sociocultural. Electronic Journal of Research in Educational Psychology, 6(16), 793-822. https://www.redalyc.org/pdf/2931/293121916012.pdf

Recio, M. (2008). Enfoques de aprendizaje y rendimiento en alumnos de educación a distancia. Revista Internacional de Ciencias Sociales y Humanidades, SOCIOTAM, 18(2), 113-144. https://www.redalyc.org/pdf/654/65411193006.pdf 
Reid, J. M. (1987). The learning style preferences of ESL Students [Las preferencias de estilo de aprendizaje de los estudiantes de ESL]. TESOL Quarterly, 21(1), 87-111. https://www.jstor.org/stable/3586356

Richardson, J. T. E. (2005). Students' approaches to learning and teachers' approaches to teaching in higher education [Los enfoques de aprendizaje de los estudiantes y los enfoques de los profesores para la enseñanza en la educación superior]. Educational Psychology, 25(6), 673-680. https://doi.org/10.1080/01443410500344720

Richardson, J. T. E. (2010). Conceptions of learning and approaches to studying among White and ethnic minority students in distance education [Concepciones de aprendizaje y enfoques de estudio entre estudiantes blancos y de minorías étnicas en educación a distancia]. British Journal of Educational Psychology, 80(4), 535-556. https://doi.org/10.1348/000709910X489283

Richardson, J. T. E. (2011). Approaches to studying, conceptions of learning and learning styles in higher education [Enfoques del estudio, concepciones de aprendizaje y estilos de aprendizaje en la educación superior]. Learning and Individual Differences, 21(3), 288293. https://doi.org/10.1016/j.lindif.2010.11.015

Rico-Reintsch, K. I. (2019). Uso de autoevaluación docente como herramienta innovadora para el mejoramiento de las asignaturas universitarias. Revista CEA, 5(10), 69-81. https://doi.org/10.22430/24223182.1445

Rivera, P., Sánchez, P., Romo, E., Jaramillo, A. y Valencia, A. (2013). Percepciones de los estudiantes universitarios frente al aprendizaje por medio de dispositivos móviles. Educación y Desarrollo Social, 7(2), 152-165. https://doi.org/10.18359/reds.687

Rodríguez, H., Pirul, J., Robles, J., Pérez, L., Vásquez, E., Galaz, I., Cuellar, C., Díaz, H., y Arriaza, C. (2018). Análisis de los estilos de aprendizaje en alumnos de Medicina de la Universidad de Chile. Educación Médica, 19(1), 2-8. https://doi.org/10.1016/j.edumed. 20 $\underline{16.11 .004}$

Rojas-Jara, C., Díaz-Larenas, C., Vergara-Morales, J., Alarcón-Hernández, P., y Ortiz-Navarrete, M. (2016). Estilos de enseñanza y estilos de aprendizaje en educación superior: Análisis de las preferencias de estudiantes de Pedagogía en Inglés en tres universidades chilenas. Revista Electrónica Educare, 20(3), 1-29. http://dx.doi.org/10.15359/ree.20-3.7 
Rosário, P., Núñez, J. C., González-Pienda, J., Almeida. L., Soares, S., y Rubio, M. (2005). El aprendizaje escolar examinado desde la perspectiva del «Modelo 3P» de J. Biggs. Psicothema, 17(1), 20-30. http://www.psicothema.com/pdf/3059.pdf

Rosário, P., Núñez, J. C., González-Pienda, J., Valle, A., Trigo, L., \& Guimarães, C. (2010). Enhancing self-regulation and approaches to learning in first-year college students: A narrative-based programme assessed in the Iberian Peninsula [Mejora de la autorregulación y los enfoques de aprendizaje en estudiantes universitarios de primer año: un programa basado en la narrativa evaluada en la Península Ibérica]. European Journal of Psychology of Education, 25(4), 411-428. https://doi.org/10.1007/s10212-010-0020-y

Santizo, J. A., García, J. L., y Gallego, D. J. (2008). Dos métodos para la identificación de diferencias de estilos de aprendizaje entre estudios donde se ha aplicado el CHAEA. Revista de Estilos de Aprendizaje, 1(1), 28-42. $\underline{\text { http://www.uned.es/ }}$ revistaestilosdeaprendizaje/numero_1/artigos/lsr_rincon_cue_gallego.pdf

Schommer, M. (1998). The influence of age and education on epistemological beliefs [La influencia de la edad y la educación en las creencias epistemológicas. Británico]. British Journal of Educational Psychology, 68(4), 551-562. https://doi.org/10.1111/j.20448279.1998.tb01311.X

Tanriverdi, B. (2012). Pre-service teachers' epistemological beliefs and approaches to learning [Creencias epistemológicas y enfoques de aprendizaje de los profesores en formación]. Procedia-Social and Behavioral Sciences, 46, 2635-2642. https://doi.org/10.1016/j.sbspro.2012.05.538

Valle, A., González, R., Núñez, J. C., Suárez, J. M., Piñeiro, I., y Rodríguez, S. (2000). Enfoques de aprendizaje en estudiantes universitarios. Psicothema, 12(3), 368-375. http://www.psicothema.com/psicothema.asp?id=344

Vergara-Hernández, C., Simancas-Pallares, M., \& Carbonell-Muñoz, Z. (2019). Psychometric properties of the revised two-factor study process questionnaire - R-SPQ-2F. Spanish version [Propiedades psicométricas del cuestionario revisado del proceso de estudio de dos factores - R-SPQ-2F. Versión en español]. Duazary, 16(2), 205-218. http://dx.doi.org/10.21676/2389783X.2744 
Winberg, T. M., Hofverberg, A., \& Lindfors, M. (2019). Relationships between epistemic beliefs and achievement goals: developmental trends over grades 5-11 [Relaciones entre creencias epistémicas y metas de logro: tendencias de desarrollo en los grados 5 a 11]. European Journal of Psychology of Education, 34(2), 295-315. https://doi.org/10.1007/s10212-018$\underline{0391-\mathrm{Z}}$

Zakariya, Y. F., Bjørkestøl, K., Nilsen, H. K., Goodchild, S., \& Lorås, M. (2020). University students' learning approaches: An adaptation of the revised two-factor study process questionnaire to Norweian [Enfoques de aprendizaje de los estudiantes universitarios: una adaptación del cuestionario revisado del proceso de estudio de dos factores al noruego]. $\begin{array}{lllll}\text { Studies in Educational } & \text { Evaluation, } & 64, & \end{array}$ https://doi.org/10.1016/j.stueduc.2019.100816 